SHS Web of Conferences 10, 00009 (2014)

DOI: $10.1051 /$ shsconf/20141000009

C Owned by the authors, published by EDP Sciences, 2014

\title{
Safe bicycling - Problems and solutions
}

\author{
M. Grecka ${ }^{1}$, A. Vilumsone ${ }^{1}$, J. Blums ${ }^{1}$, and Z. Pavare ${ }^{2}$ \\ ${ }^{1}$ Riga Technical University, Latvia \\ ${ }^{2}$ Rīga Stradiņš University, Latvia
}

\begin{abstract}
Herewith a popularity of bicycling, various safety problems of cyclists are researched. Studies are connected with infrastructure, riding culture, choice of cyclist's suit and riding equipment. Overall, 382 respondents (159 cyclists, 119 drivers, 104 pedestrians) were involved in the survey. The road participants' mutual communication problems in the traffic and opportunities of solving them were clarified.

Most of cyclists wear comfortable daily or sporty clothing with reflective elements, and in their opinion, clothing with light emissive elements must be like daily clothing. The drivers could better notice cyclists, if they wear brightly colored suit with light emissive and reflective elements, but pedestrians - if cyclists use warning sound signals. The opinions of road participants about the placement of light emissive and reflective elements in cyclist's clothing were clarified.

The prototype of cycling belt (Fig. 1e) has been drawn up with LED stop signals, which are activated by the accelerometer placed in the central back pocket. The results of approbation have shown that the sensor does not provide the proper functioning of signals, due to a high level of riding movements. Using Motion Capture technology and bicycle exercise equipment, the research of oscillation of anthropometric points on the back has been carried out. The accelerometer should be placed between scapulae to design the cycling jacket.
\end{abstract}

\section{Introduction}

Bicycle is considered a beneficial transport and cycling is 5 times more effective than any type of travelling, for example, hiking, or distance walking. Herewith an increasing number of cyclists, the safety problems of cyclists are researched [4]. These problems are related with many aspects, e.g. cycling infrastructure, the riding culture of the road participants and choice of corresponding clothing [1, 3-5]. The cyclist's suit is defined by rider's style, the type of bicycle, the functional features and construction of clothing, and also by the use of light reflective (LR) and emissive elements (LE) (Fig. 1). These elements can remarkably improve cyclists' visibility during day and night time and also can be used as devices for communication with car drivers and other cyclists.

LR elements are widely used in brightly colored upper clothing (like inlets or patches) and accessories - different covers, straps, belts, bags. The usage of LE elements in cyclist's suit is not so popular yet as usage of reflective ones, although they become more and more popular, e.g., designers' clothing, popular sport brands, and also among professional and amateur riders and inventors.

This is an Open Access article distributed under the terms of the Creative Commons Attribution License 4.0, which permits unrestricted use, distribution, and reproduction in any medium, provided the original work is properly cited. 


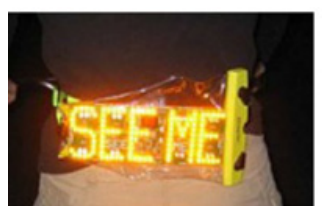

a

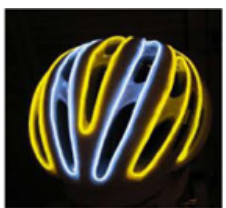

b

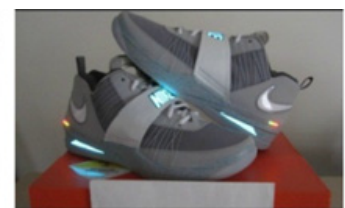

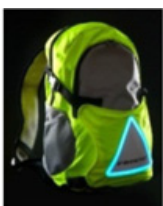

d

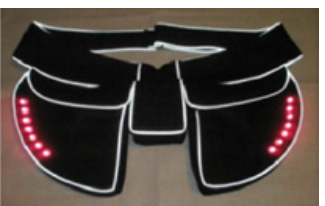

Figure 1. Light emissive elements in sport suit: a - a belt with LED text [9], b - a bicycle helmet with EL wires [7], c - boots with EL strips (Nike) [11], bag with reflective and EL strip [13], smart cyclist's belt with LR and LEDs.

\begin{tabular}{|l|}
\hline \multicolumn{2}{|c|}{ The Groups of Respondents } \\
\hline \multicolumn{1}{|c|}{ Cyclists } \\
\hline $\begin{array}{l}\text { Car drivers } \\
\text { Problems (accidents) on roads (pavements) with road participants }\end{array}$ \\
\hline \hline $\begin{array}{l}\text { Length of cycling/driving; } \\
\text { Placement, necessity and functionality of LR and LE } \\
\text { elements in cyclists' clothing }\end{array}$ \\
\hline \hline $\begin{array}{l}\text { Type of bicycle, } \\
\text { clothing; }\end{array}$ \\
\hline
\end{tabular}

Figure 2. Distribution of theme of question(s).

The prototype of cycling belt (Fig. 1e) with pockets and light emissive elements has been developed within the framework of the present research. The principle of the belt is: while the cyclist is riding at a constant speed or is at rest state, the LEDs flash, but when the cyclist is braking the LEDs light up instantly (like brake lights), which are activated by a signal coming from the sensor (accelerometer), placed in the central back pocket.

The approbation has proven that the sensor (placed on the back of the belt) does not provide the proper functioning of stop signals, due to the high level of additional movements of a cyclist during the riding. As in this case it is unacceptable, the research of oscillation of the anthropometrical points on the back has been carried out to clarify the most appropriate placement of accelerometer for the design of cycling jacket (Fig. 5). Within the framework of the research, bicycle exercise equipment for cycling imitation and Optical Active Motion Capture technology have been used [10].

Motion Capture technology consists of several types, therefore it has become quite popular in biomechanics [10]. The technology is used for bicycle fitting (e.g. distance between the saddle and the handlebar, the distance between the pedals) for the rider and also for optimization of positions of rider's body extremities, thus improving riding technique, reaching better results and avoiding injuries of joints and muscles $[12,8]$. Some studies include research of motion of separate body parts depending on their positions and conditions, e.g., pelvis motion or thorax during sustained cycling [14, 15].

The aim of the present study - to clarify the road participants' mutual communication problems in the traffic and opportunities of solving them with integrating light reflective and emissive elements in 
Int. Conf. SOCIETY. HEALTH. WELFARE.

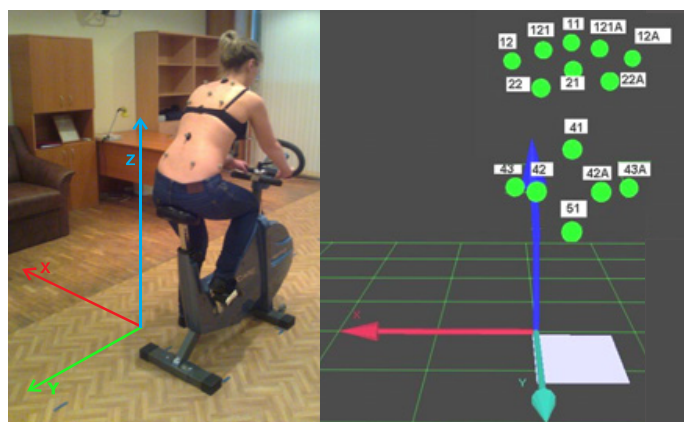

a

b

Figure 3. Placement of the markers; axis of coordinates and markers in real (a) and virtual (b) environment.

cyclist's suit, and also to define the placement of electronic components for the activity of projected clothing.

\section{Materials and methods}

\section{Sociological survey}

Sociological survey was carried out using a form of web questionnaires. Overall 382 respondents or three groups of road participants were involved in the survey (159 cyclists, 119 drivers and 104 pedestrians). Questionnaires for each group contained common and different questions. Cyclists were asked more questions than other two groups. Questions were about their bicycle type and cycling aim, clothing and its features, about attitude and communication problems with car drivers and other road participants. Pedestrians and car drivers were asked about attitude to cyclists and communication problems with them. Opinions about placement, necessity and functionality of light reflective and light emissive elements were asked to both cyclists and drivers. All groups were asked questions about colours of cyclists' suit, gender and age.

\section{B Motion capture}

The motion capture technology has been used in the study. The digital cameras of the installed system recorded the movement of anatomical points in 3 dimensions [6]. To record the motion, 14 markers (small spheres, which are detected with infrared cameras) have been placed and attached to definite human anatomical points of the body (Fig. 3). The points have been marked with numbers and letters according to the points, which are used in construction of clothing.

The least mobile point of the back was identified during riding a bicycle at different speeds $(15 \mathrm{~km} / \mathrm{h}$, $20 \mathrm{~km} / \mathrm{h}, 25 \mathrm{~km} / \mathrm{h}$, increasing from 15 to $30 \mathrm{~km} / \mathrm{h}$ ) and in 2 positions of the bodice. Position "a“- hands are on the lower part of the handlebars and position " $b$ " - hands are on the upper part of the handlebars.

Six infrared light cameras sent an infrared beam $(120 \mathrm{~Hz})$ and detected reflection of spherical markers. The motion trajectories of the back points in 3 dimensions were registered, and coordinates in time of reflective markers were calculated.

Eight experimental "rides", per 10 seconds, were made at different speeds and in two slopping positions (A and B) of the body. During the experiment markers 42, 43, 42A, 43A (placed on the lower part of the back) were excluded from the further calculations, due to their greater amplitude of motion than other markers. 
Total amplitude of acceleration of each marker (2) was clarified, when minimal and maximal values of total acceleration were identified. The component of acceleration (1) of motion point in the definite direction is the derivation of component of speed, or the second derivation of corresponding coordinate [2]:

$$
a_{x}=\frac{d v_{x}}{\mathrm{dt}}=\frac{d^{2} x}{d t^{2}} a_{y}=\frac{d v_{y}}{\mathrm{dt}}=\frac{d^{2} y}{d t^{2}} a_{z}=\frac{d v_{z}}{\mathrm{dt}}=\frac{d^{2} z}{d t^{2}},
$$

where $a_{x}-x$ component of the acceleration;

$a_{y}-y$ component of the acceleration;

$a_{z}-z$ component of the acceleration;

$t$ - time (s);

$v_{x}-x$ component of the velocity;

$v_{y}-y$ component of the velocity;

$v_{z}-z$ component of the velocity.

Absolute value of acceleration is:

$$
a_{\text {total }}=\sqrt{a_{x}^{2}+a_{y}^{2}+a_{z}^{2}},
$$

where $a_{\text {total }}$ - the module of total acceleration.

\section{Results}

\section{A. Results}

Majority of cyclists ride a mountain bike in the city at daytime during the period of spring-autumn. Most often, the bicycles are used for active recreation, riding to/from studies/work and daily rides. Cyclists wear comfortable daily or sporty clothing with reflective inlets and patches.

Although the majority of riders' bicycles are equipped according to the rules of traffic (white light or reflector in the front, red light or reflector in the back, orange reflectors on wheels) part of the cyclists happened to be involved in road accidents. In cyclist's opinion, the features of clothing with light emissive elements must be analogical with daily ones. Mostly used cyclists' accessories are gloves and rucksacks, and reflective elements mostly are used in jackets.

A half of the questioned drivers treat the cyclists as equal value road participants. Not using reflective elements on cyclists' clothing and cyclists' and manoeuvring without any prior warning signals cause problems for drivers, when they are in active traffic. Drivers can better see cyclists if they wear brightly colored clothing with light reflective and emissive elements.

Most of pedestrians live in the city, where they meet cyclists frequently. Only quarter of pedestrians collided with cyclists on pavement. Pedestrians will better notice the cyclists, if they use sound signals.

All the groups of respondents consider that the most suitable colours for cyclists' clothing are yellow and orange for upper part (jacket, shirt etc.), and black, yellow and orange for lower part (trousers, shorts).

Road participants' opinions about the placement of light emissive and reflective elements in cyclist's clothing were clarified (Table).

\section{B. Results}

The lowest amplitude of acceleration in position A (Fig. 4a) at different speeds is characteristic of markers 22, 22A, 21, but the largest - of markers 121, 121 A, 12A. The lowest amplitude of acceleration 
Int. Conf. SOCIETY. HEALTH. WELFARE.

Table 1. Placement of light emissive and reflective elements in cyclist's clothing.

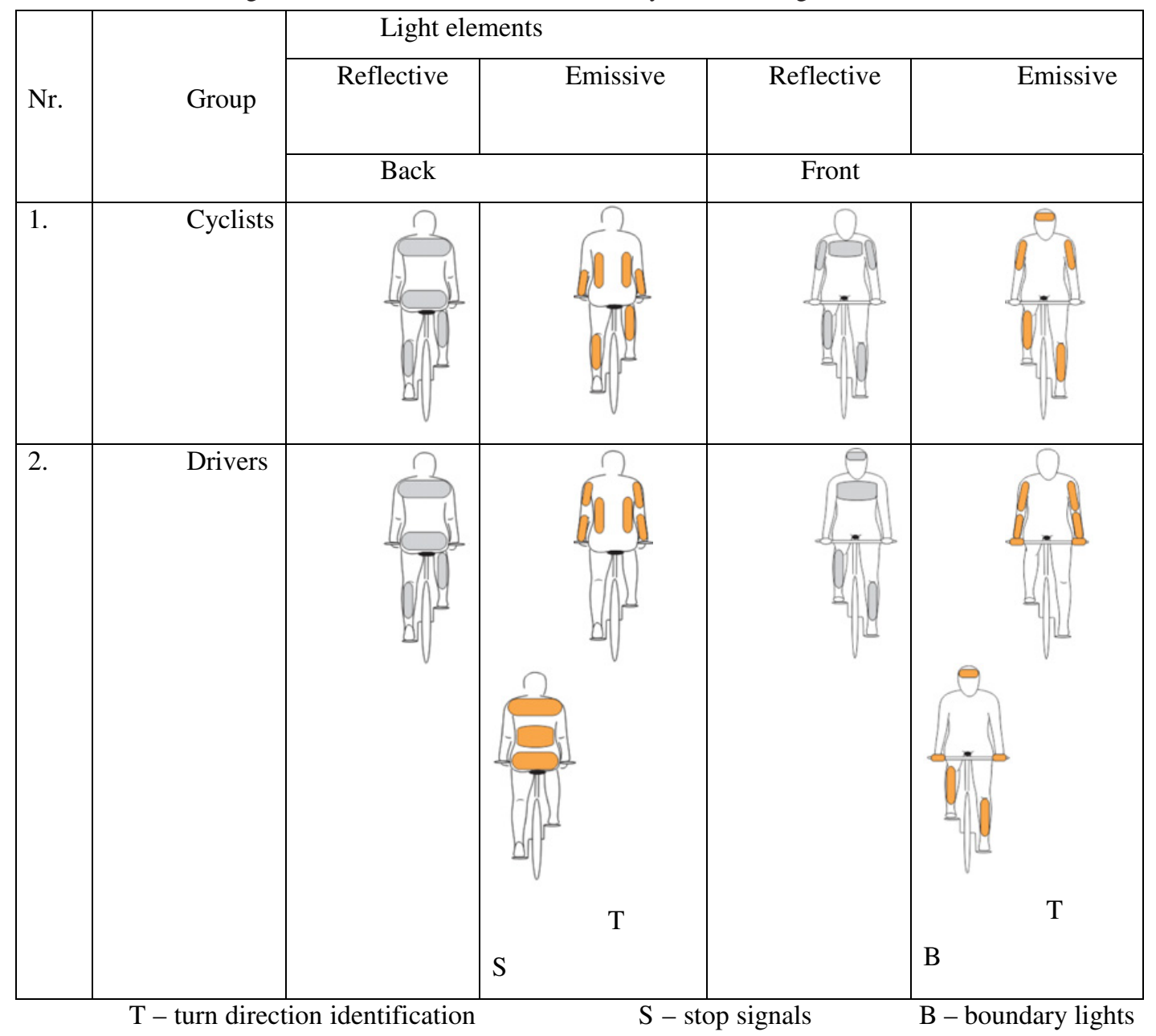
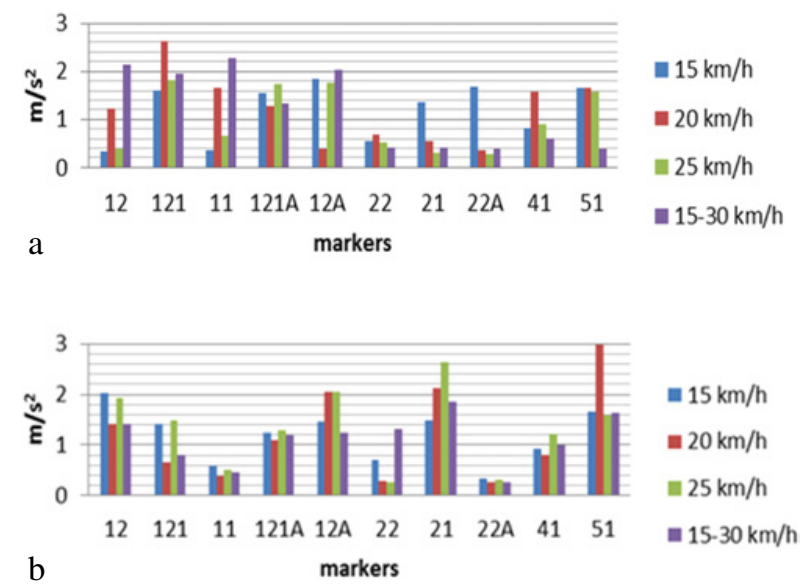

Figure 4. Amplitude of total acceleration of markers in position A (a) and position B (b). 


\section{SHS Web of Conferences}

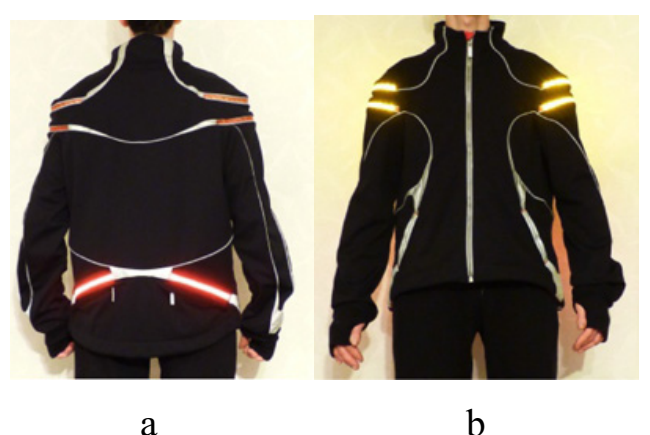

a

b

Figure 5. Cyclist's jacket, back part (a), front part (b).

in position B (Fig. 4b) at different speeds is characteristic of markers 11, 22, 22A, but the largest - of markers $12,12 \mathrm{~A}, 21,51$.

\section{Discussion}

\section{A}

Mostly the cyclists prefer riding at daytime during warm period of time (spring, summer, autumn). As bicycles are used for active recreation, riding to/from studies/work and daily rides, for each of this type of ride special clothing is needed. For example, for recreation the rider will use sporty clothing, but for studies/work - daily or even work clothing. In this case, developed cyclist's belt (Fig. 1e) can be used as for ridings to studies/work or in hot summer days, but cyclist's jacket - for active recreation/daily rides or cooler days.

Although the majority of riders' bicycles are equipped according to the rules of traffic, part of cyclists happened to be involved in road accidents, which can be caused by lack of cycling tracks and riding culture of cyclists and car drivers.

Cyclists can be better noticed by car drivers in day time, if their clothing is bright, but at dark time, if their clothing is equipped with blinking light elements, which draw drivers' attention like other lights of cars.

Not all bicycles have sound warning signals, thereby, many pedestrians, who walk on pavement, do not notice the cyclist, who rides from the back or one of the side, and get into unpleasant accident.

\section{B}

The goal of the study has been to detect the points of the back, whose periodical motions, during the pedalling, minimally influence the readouts of accelerometer and allow them, without disturbances, to define the changes in speed in the riding direction of bicycle. Experimental researches demonstrate that the motion dynamics of markers depends on the position of the bodice and riding speed. Therefore the most suitable place for accelerometer is the body point 21 or 11 , or between them.

From the point of view of the clothing construction these points are acceptable, as they can be placed on the back central seam. The sensor can be easily integrated, placing it on seam allowances (sensor parameters $10 \times 15 \times 2 \mathrm{~mm}$ ). The detail (-s) of clothing upper part of the back is less influenced by deformation.

In terms of safety, considering anthropometric features, the sensor at this place (marker 21) is less influenced mechanically, as there is concavity between scapulae. For example, putting on a rucksack, the sensor will not be damaged. 


\section{Int. Conf. SOCIETY. HEALTH. WELFARE.}

Taking into account the answers and opinions of road participants, and research of placement of electronic components, the cyclist's jacket with light emissive elements in the shoulder part and back, and with light reflective patches, edges and inlets in the constructive seams was developed (Fig. 5). The main function of the jacket improves cyclist's safety in the active traffic and enhances communication with other road users. It is supported by several auxiliary functions: stop signal, when cyclist is braking; the right/left turn indicator and emergency lights activation by integrated buttons in the cuffs; light reflective elements which highlight the cyclist's silhouette.

\section{Conclusions}

For safe cycling not only the infrastructure of bicycling must be well equipped, but also special clothing must be developed, which can remarkably improve cyclists' visibility at any day time and enable better communication with other road participants. In that way, a smart cyclist's jacket with light reflective elements, direction turning identification and stop signals was developed.

\section{References}

[1] Anonymous (2010). The promotion of cycling, Directorate-general for internal policies, Policy department, Structural and cohesion policies. Brussels: (CEuropean Parliament

[2] Apinis, A.: Fizika (Physics), Zvaigzne, Rīga (1972). (in Latvian)

[3] Basford L.R., Lester S., Thomson T. (2002). Driver's perceptions of cyclists

[4] Buehler, R. (2009). Cycling for a Few or for Everyone: The Importance of Social Justice in Cycling Policy. World Transport Policy and Practice, 15(1), 57-64

[5] Christmas S., Helman S., ed. (2010). Cycling, Safety and Sharing the Road: Qualitative Research with Cyclists and Other Road Users, Road Safety Publication No. 17, Research Laboratory. London: Department for Transport, September 2010

[6] Dabolina, I. Vilumsone, A. Fjodorovs, A. (2009). Application of Non-Contact 3D Positioning for Anthropometrical Modelling, Scientific Journal of RTU, vol. 4, 138-145

[7] Enlighted (author), "Instructables", How to add EL wire to a coat or other garment, June, 2008 [Online]. Available: www.instructables.com/id/how-to-add-EL-wire-to-a-coat-orother-garment/step10/other-examples-EL-wire-hats-and-helmets/

[8] Fitwerx, The future of bike fitting, 2011 [Online]. Available: http://www.fitwerx.com/motioncapture-analysis

[9] Fritz Y., "Cyclelicious”, August 2009 [Online]. Available: http://www.cyclelicio.us/2009/08/seeme-light-belt.html

[10] Lee J., Home page - professor Jehee Lee, courses, April, 2011 [Online]. Available: http://mrl.snu.ac.kr/courses/CourseAnimation/notes/MotionCapture.pdf

[11] Nice Kicks [Online]. Available: http://www.nicekicks.com/2013/04/26/nike-zoom-revis-magcustom-2/

[12] Retul, Retul Fit Technology, 2012 [Online]. Available: http://retul.com/about-retul.asp [Accessed: Sep. 15, 2012]

[13] Running supplement [Online]. Available: http://www.runningsupplement.co.uk/reviews/ HighViz/vizpro.html

[14] Sauer J., Potter J. (2007). Influence of gender, power, and hand position on pelvic motion during seated cycling. Medicine and Science in Sports and Exercise, 39(12), 2204-2211

[15] Sayers M., Tweddle A. (2012). Thorax and pelvis kinematics change during sustained cycling. International Journal of Sports Medicine. 33(4), 314-319 